\title{
Improving Language Acquisition and Processing With Cognitive Stimulation
}

\author{
José Luis Tapia ${ }^{1}$ and Jon Andoni Duñabeitia ${ }^{1,2 *}$ \\ ${ }^{1}$ Centro de Ciencia Cognitiva (C3), Universidad Antonio de Nebrija, Madrid, Spain, ${ }^{2}$ AcqVA Aurora Center, The Arctic \\ University of Norway, Tromsø, Norway
}

Keywords: cognitive stimulation, brain training, language learning, executive functions, intervention programs

\section{INTRODUCTION}

Cognitive functions are essential in human development in general, and they play a key role in language learning, as well as in reading and writing. A large body of evidence makes the relationship between executive functions and language acquisition and processing indisputable [Moser et al., 2007; Mazuka et al., 2009; Woodard et al., 2016; see also the meta-analysis by Swanson et al. (2009)]. Lexical-semantic processing has been associated with inhibition skills (Khanna and Boland, 2010) and with working memory and information updating (Weiland et al., 2014), whereas syntactic processing has been linked with inhibition, shifting, updating (Novick et al., 2005; Roberts et al., 2007). Memory updating has been suggested to underlie both sentence comprehension (Daneman and Carpenter, 1980) and production (Slevc, 2011). Furthermore, executive functions have also been correlated with the development of phonological awareness (Risso et al., 2015). Broadly speaking, the neuroscientific literature has consistently shown that executive functions and

OPEN ACCESS

Edited by:

Cristina Baus,

Pompeu Fabra University, Spain

Reviewed by:

Marco Calabria,

Open University of Catalonia, Spain

*Correspondence: Jon Andoni Duñabeitia jdunabeitia@nebrija.es

Specialty section:

This article was submitted to Language Sciences, a section of the journal Frontiers in Psychology

Received: 03 February 2021 Accepted: 19 April 2021 Published: 14 May 2021

Citation:

Tapia JL and Duñabeitia JA (2021) Improving Language Acquisition and Processing With Cognitive Stimulation.

Front. Psychol. 12:663773.

doi: $10.3389 /$ fpsyg.2021.663773 language skills are interrelated, suggesting an overlap of the neural processes involved [see Slot and Von Suchodoletz (2018)].

Despite the large body of research demonstrating the close link between executive functions and language skills, it is yet to be established the possible bidirectionality or reciprocality between the development of both macro-systems and the associated skills. Following the notion of brain plasticity and the expansion-partial renormalization hypothesis (EPH) (Pliatsikas, 2020), any new cognitive effort, such as acquiring a new language, may produce a change in the neural system and pathways (e.g., increasing the number of synapses, generating new dendritic spines, or strengthening neural connections) related to this learned skill. With this being so, and using digital biomarkers, one could potentially quantify the specific changes in the cognitive system induced by language learning, but more importantly, one could also determine the best cognitive foundations on which language learning could be built by virtue of establishing the reciprocal connections between domain-general executive functions and language acquisition. Furthermore, as RojasBarahona et al. (2015) proposed, a cognitive stimulation intervention focused on these biomarkers could potentially increase and strengthen the neural network underlying language skills.

\section{COGNITIVE STIMULATION AND BRAIN TRAINING: CONCEPT AND EFFECTIVENESS}

Although cognitive stimulation, cognitive training, and brain training may represent interchangeable terms, a correct nomenclature should be adopted to correctly narrow down the extent to which a given intervention is expected to impact cognitive behavior. Cognitive stimulation typically refers to all those techniques and strategies that aim to improve the cognitive functioning of different capacities and cognitive functions such as attention, reasoning, memory, 
perception, abstraction, language, or praxis. Cognitive stimulation seems to be more appropriate than the other terms, since these interventions do not really "train" the brain as an organ but stimulate its activity and the cognitive functions. Hence, cognitive stimulation stands as an all-encompassing umbrella term that could adequately account for the different intervention protocols aimed to improve cognitive functions and/or the underlying neural bases and mechanisms.

In the 1970s, clinical intervention programs were designed aimed at the restoration of damaged cognitive functions focusing mainly on the cognitive domains of attention, executive functions, working memory, processing speed, and reasoning. A large number of therapists began to use cognitive training as a path to neuropsychological rehabilitation of patients with brain injury (Sohlberg and Mateer, 1987), depression (Zeiss et al., 1979), cognitive impairment (Labouvie-Vief and Gonda, 1976), hyperactivity (Douglas et al., 1976), or schizophrenia (Olbrich and Mussgay, 1990). These protocols soon began to be criticized given the presence of methodological shortcomings. As Abikoff (1979) reviewed, in most cases, interventions for cognitive rehabilitation resulted in positive outcomes in the directly trained functions, with none or limited transferability to other cognitive skills or everyday functioning [see also Wilson (1997)].

Early in the twenty-first century, the market around commercial cognitive stimulation programs started to become increasingly prominent, reigniting the interest in the field. Since 1999, when a group of scientists and academics created a company to promote cognitive training and launched CogniFit (CogniFit Inc., San Francisco, US), many other companies have created similar products with different degrees of success (e.g., Cogmed, now property of Pearson Education, London, UK; BrainHQ by Posit Science Corporation, San Francisco, US). In 2005 the Japanese market revolutionized the field when Brain Age (Nintendo Co. Ltd., Kyoto, Japan) was first launched, and it was soon extended to the American and European market in 2006. Due to the rise and spread of these programs, the US Federal Trade Commission started to arbitrate the market, sueing some companies for deceptive advertising, and giving rise to an agitated period in which the effectiveness of cognitive stimulation programs was questioned [see Allaire et al. (2014), for a letter claiming for inflated and misleading results because of a longstanding industry behind these products; but see Alescio-Lautier et al. (2014), for a response letter in defense of the efficacy of cognitive stimulation programs].

Over the years, cognitive stimulation has been used in a wide variety of areas, such as learning and education (MelbyLervåg and Hulme, 2013), psychological disorders (LawlorSavage and Goghari, 2014), brain damage (Spreij et al., 2014) or neurodegenerative disorders (Reijnders et al., 2013), reporting improvements in overall cognition and in specific cognitive domains in healthy and unhealthy samples [see Wang et al. (2016)]. In this vein, cognitive stimulation programs have adopted multiple forms and approaches, ranging from scientific interventions based on neuroplasticity (Nahum et al., 2013) and cognitive constructs (Jaeggi et al., 2008) or approaches inspired by meditation practices (Tang et al., 2007), to more gamified interventions (Anguera et al., 2013), or to commercial videogames originally designed for other purposes (Green and Bavelier, 2003). This diversity of target populations and approaches has been partially responsible for the controversy on the reproducibility and generalizability of the results obtained across studies and on the effectiveness of cognitive stimulation programs [see Fisher (2014)].

The effectiveness of an intervention based on a cognitive stimulation program could be measured from two viewpoints. On the one hand, a cognitive stimulation program could be considered effective as long as the specific function worked significantly improves between a pre-intervention evaluation and a post-intervention assessment. On the other hand, the effectiveness of a cognitive stimulation program could be also understood as the improvements found in general cognitive functioning -far effects- and not only in the trained domain -near effects- (Lindenberger et al., 2017). According to this last view, for an intervention to be effective, improvements should be also found in untrained tasks and daily functioning (Simons et al., 2016). Most of the evidence obtained in cognitive stimulation interventions has focused on improvements in the tasks or domains directly worked (i.e., near transfer effects; see van Heugten et al., 2016, for review), but far transfer effects have been also reported, although to a lesser degree [see Dahlin et al. (2008), Hardy et al. (2015); see Au et al. (2015), for a meta-analysis].

\section{OPTIMIZATION OF COGNITIVE STIMULATION PROGRAMS}

The most relevant quest to tackle in cognitive stimulation research is precisely how to achieve far transfer effects. Here we propose three different strategies that could be combined to foster generalization to other untrained mechanisms or skills: the ecological validity and capacity of adherence of the intervention, the length and spacing of the training and its sessions, and the dynamic adaptation of the program to the cognitive state of each individual at each stage of the process.

First, we would like to stress out the importance of designing ecologically relevant and motivating protocols. Training a specific skill or even a specific cognitive domain will presumably bring off improvements in those areas. However, in order to find an impact on daily operations and functioning, intervention programs would necessarily contemplate everyday life problems and life-like tasks, including social interactions (Engert et al., 2017; Valk et al., 2017). Besides, motivation may function as a hidden factor masking far transfer effects and modulating adherence and dropout rates. Participants' expectations about a given intervention's outcome and their subsequent motivation toward it can drastically change the observed effects (Finniss et al., 2010; Rutherford et al., 2010; Boot et al., 2013; Keitel et al., 2013).

Second, the establishment of a scientifically validated training timespan needed to obtain far transfer effects is needed. While in some studies participants train for a short period of time every day, in others they train only some days a week, showing great variability in the training time from one study to another. Besides, 
the structure of some cognitive stimulation programs lasts for weeks (e.g., Knapp et al., 2006), while others can last for years.

And third, the natural variability associated with baseline performance across the set of the cognitive domains or abilities that will be trained is a critical factor that could modulate the effectiveness of any intervention. In other words, the interindividual differences of two trainees with different baseline levels of cognitive skills and different improvement paces would require that cognitive stimulation programs adjust the level of complexity or difficulty of the set of tasks to offer tailormade interventions. While some programs present seemingly scalar levels of difficulty that statically adapt to certain initial user profiles, the key aspect is the inclusion of fully adaptive algorithms that adjust the difficulty levels and intensity of the training dynamically throughout the process. Hence, the dynamic adaptation of any cognitive stimulation program to the cognitive baseline of each trainee stands as an essential requisite to foster not only maximization of the benefits of the training, but also adherence to it.

\section{DISCUSSION}

New technologies make it possible to computerize cognitive stimulation programs in a likable gaming environment. The computerization of the tasks and the development of adaptive difficulty algorithms allow the design of challenging activities that require dynamically changing levels of cognitive effort, yielding an enhancement of the outcomes. This, together with the possibility of adjusting the protocols thanks to the large amount of data collected through the same intervention program across persons and devices, increases the chances to develop data-informed ecologically valid adaptative cognitive stimulation programs and platforms (see the products of CogniFit, as a paradigmatic example).

The brain changes and cognitive adaptation that occur when a person acquires a new language suggest that language could be considered as one of the most natural brain training programs for enhancing cognitive functions [see Luk et al. (2020), Pliatsikas (2020), for review]. Not surprisingly, in the last decade a whole line of research has focused on the impact of multilingualism or of the acquisition of a non-native language in domain-general

\section{REFERENCES}

Abikoff, H. (1979). Cognitive training interventions in children: review of a new approach. J. Learn. Disabil. 12, 123-135. doi: 10.1177/0022219479012 00213

Alescio-Lautier, B., Allen, M., Andersen, R. A., Ball, K. K., Banai, K., Baniel, A., et al. (2014). Cognitive Training Data Response Letter. Available online at: https://www.cognitivetrainingdata.org/the-controversy-does-braintrainingwork/response-letter/ (accessed November 18, 2020).

Allaire, J. C., Bäckman, L., Balota, D. A., Bavelier, D., Bjork, R. A., and Bower, G. H. (2014). A Consensus on the Brain Training Industry from the Scientific Community. Stanford Center on Longevity, Max Planck Institute for Human Development. Available at http://longevity3.stanford.edu/blog/2014/10/15/theconsensus-on-the-brain-training-industry-from-the-scientific-community2/ (accessed January 31, 2021). cognitive skills [see Antón et al. (2019), Leivada et al. (2021)]. But over and above the possibility of exploring and measuring the cognitive milestones attained in the process of language training, it is worth considering also a different approach resulting from taking the opposite view angle. Given the close link between domain-general sets of cognitive skills such as executive functions and language learning, one could tentatively predict that interventions based on cognitive stimulation programs could give rise to enhanced language learning. Put differently, given that learning a second or foreign language could result in far transfer effects in non-linguistic cognitive skills, it could also be expected that specific non-linguistic cognitive training could improve not only the cognitive skills that underlie language control [see Liu et al. $(2016,2019)$ ], but also boost language learning and linguistic skills. While this is a very incipient line of research that is still in its infancy, a handful of studies already bring hope to this approach (e.g., Hayashi, 2019; Karousou and Nerantzaki, 2020).

Interventions based on cognitive stimulation programs have been carried out effectively in all age ranges, both in persons with different pathologies or cognitive dysfunctions and in healthy individuals. With this in mind and taking into account that the largest portion of the world speaks two languages or is learning a non-native language, the inclusion of cognitive stimulation programs in the daily agenda of current and future multilinguals seems a logical step.

\section{AUTHOR CONTRIBUTIONS}

All authors listed have made a substantial, direct and intellectual contribution to the work, and approved it for publication.

\section{FUNDING}

This research has been partially funded by grants PGC2018097145-B-I00 and RED2018-102615-608-T from the Spanish Government and H2019/HUM-5705 from the Comunidad de Madrid. The funders had no role in study design, data collection and analysis, decision to publish, or preparation of the manuscript.
Anguera, J. A., Boccanfuso, J., Rintoul, J. L., Al-Hashimi, O., Faraji, F., Janowich, J., et al. (2013). Video game training enhances cognitive control in older adults. Nature 501, 97-101. doi: 10.1038/nature12486

Antón, E., Carreiras, M., and Duñabeitia, J. A. (2019). The impact of bilingualism on executive functions and working memory in young adults. PLoS ONE 14:e0206770. doi: 10.1371/journal.pone.02 06770

Au, J., Sheehan, E., Tsai, N., Duncan, G. J., Buschkuehl, M., and Jaeggi, S. M. (2015). Improving fluid intelligence with training on working memory: a meta-analysis. Psychon. Bull. Rev. 22, 366-377. doi: 10.3758/s13423-014-0 699-x

Boot, W. R., Simons, D. J., Stothart, C., and Stutts, C. (2013). The pervasive problem with placebos in psychology: why active control groups are not sufficient to rule out placebo effects. Perspect. Psychol. Sci. 8, 445-454. doi: $10.1177 / 1745691613491271$ 
Dahlin, E., Neely, A. S., Larsson, A., Bäckman, L., and Nyberg, L. (2008). Transfer of learning after updating training mediated by the striatum. Science 320, 1510-1512. doi: 10.1126/science.1155466

Daneman, M., and Carpenter, P. A. (1980). Individual differences in working memory and reading. J. Mem. Lang. 19:450. doi: 10.1016/S0022-5371(80)90312-6

Douglas, V. I., Parry, P., Marton, P., and Garson, C. (1976). Assessment of a cognitive training program for hyperactive children. J. Abnorm. Child Psychol. 4, 389-410. doi: 10.1007/BF00922535

Engert, V., Kok, B. E., Papassotiriou, I., Chrousos, G. P., and Singer, T. (2017). Specific reduction in cortisol stress reactivity after social but not attentionbased mental training. Sci. Adv. 3:e1700495. doi: 10.1126/sciadv.1700495

Finniss, D. G., Kaptchuk, T. J., Miller, F., and Benedetti, F. (2010). Biological, clinical, and ethical advances of placebo effects. Lancet 375, 686-695. doi: 10.1016/S0140-6736(09)61706-2

Fisher, B. C. (2014). The benefits of cognitive stimulation or training/rehabilitation upon brain function as an efficacious treatment for diagnosed dementia or mild cognitive decline. J. Alzheimers Dis. Parkinsonism 4, 1-3. doi: 10.4172/2161-0460.1000161

Green, C. S., and Bavelier, D. (2003). Action video game modifies visual selective attention. Nature 423, 534-537. doi: 10.1038/nature01647

Hardy, J. L., Nelson, R. A., Thomason, M. E., Sternberg, D. A., Katovich, K., Farzin, F., et al. (2015). Enhancing cognitive abilities with comprehensive training: a large, online, randomized, active-controlled trial. PLOS ONE 10:e134467. doi: 10.1371/journal.pone.0134467

Hayashi, Y. (2019). Investigating effects of working memory training on foreign language development. Mod. Lang. J. 103, 665-685. doi: 10.1111/modl.12584

Jaeggi, S. M., Buschkuehl, M., Jonides, J., and Perrig, W. J. (2008). Improving fluid intelligence with training on working memory. Proc. Natl. Acad. Sci. U.S.A. 105, 6829-6833. doi: 10.1073/pnas.0801268105

Karousou, A., and Nerantzaki, T. (2020). Phonological memory training and its effect on second language vocabulary development. Second Lang. Res. doi: 10.1177/0267658319898514. [Epub ahead of print].

Keitel, A., Ferrea, S., Südmeyer, M., Schnitzler, A., and Wojtecki, L. (2013). Expectation modulates the effect of deep brain stimulation on motor and cognitive function in tremor-dominant Parkinson's disease. PLoS ONE 8:e81878. doi: 10.1371/journal.pone.0081878

Khanna, M. M., and Boland, J. E. (2010). Children's use of language context in lexical ambiguity resolution. Q. J. Exp. Psycholo. 63, 160-193. doi: 10.1080/17470210902866664

Knapp, M., Thorgrimsen, L., Patel, A., Spector, A., Hallam, A., Woods, B., et al. (2006). Cognitive stimulation therapy for people with dementia: cost-effectiveness analysis. $\mathrm{Br}$. J. Psychiatry 188, 574-580. doi: 10.1192/bjp.bp.105.010561

Labouvie-Vief, G., and Gonda, J. N. (1976). Cognitive strategy training and intellectual performance in the elderly. J. Gerontol. 31, 327-332. doi: 10.1093/geronj/31.3.327

Lawlor-Savage, L., and Goghari, V. M. (2014). Working memory training in schizophrenia and healthy populations. Behav. Sci. 4, 301-319. doi: 10.3390/bs4030301

Leivada, E., Westergaard, M., Duñabeitia, J. A., and Rothman, J. (2021). On the phantom-like appearance of bilingualism effects on neurocognition:(How) should we proceed?. Bilingualism 24, 197-210. doi: $10.1017 /$ S1366728920000358

Lindenberger, U., Wenger, E., and Lövdén, M. (2017). Towards a stronger science of human plasticity. Nat. Rev. Neurosci. 18, 261-262. doi: 10.1038/nrn.2017.44

Liu, C., Yang, C. L., Jiao, L., Schwieter, J. W., Sun, X., and Wang, R. (2019). Training in language switching facilitates bilinguals' monitoring and inhibitory control. Front. Psychol. 10:1839. doi: 10.3389/fpsyg.2019.01839

Liu, H., Liang, L., Dunlap, S., Fan, N., and Chen, B. (2016). The effect of domain-general inhibition-related training on language switching: an ERP study. Cognition 146, 264-276. doi: 10.1016/j.cognition.2015.10.004

Luk, G., Pliatsikas, C., and Rossi, E. (2020). Brain changes associated with language development and learning: a primer on methodology and applications. System 89:102209. doi: 10.1016/j.system.2020.102209

Mazuka, R., Jincho, N., and Oishi, H. (2009). Development of executive control and language processing. .Linguistics Compass 3, 59-89. doi: 10.1111/j.1749-818X.2008.00102.x
Melby-Lervåg, M., and Hulme, C. (2013). Is working memory training effective? A meta-analytic review. Dev. Psychol. 49:270. doi: 10.1037/a0028228

Moser, D. C., Fridriksson, J., and Healy, E. W. (2007). Sentence comprehension and general working memory. Clin. Linguist. Phon. 21, 147-156. doi: 10.1080/02699200600782526

Nahum, M., Lee, H., and Merzenich, M. M. (2013). Principles of neuroplasticity-based rehabilitation. Prog. Brain Res. 207, 141-171. doi: 10.1016/B978-0-444-63327-9.00009-6

Novick, J. M., Trueswell, J. C., and Thompson-Schill, S. L. (2005). Cognitive control and parsing: reexamining the role of Broca's area in sentence comprehension. Cognitive Affect. Behav. Neurosci. 5, 263-281. doi: 10.3758/CABN.5.3.263

Olbrich, R., and Mussgay, L. (1990). Reduction of schizophrenic deficits by cognitive training: an evaluative study. Eur. Arch. Psychiatry Neurol. Sci. 239, 366-369. doi: 10.1007/BF01734543

Pliatsikas, C. (2020). Understanding structural plasticity in the bilingual brain: the dynamic restructuring model. Bilingualism 23, 459-471. doi: $10.1017 /$ S1366728919000130

Reijnders, J., van Heugten, C., and van Boxtel, M. (2013). Cognitive interventions in healthy older adults and people with mild cognitive impairment: a systematic review. Ageing Res. Rev. 12, 263-275. doi: 10.1016/j.arr.2012.07.003

Risso, A., García, M., Durán, M., Brenlla, J. C., Peralbo, M., and Barca, A. (2015). Un análisis de las relaciones entre funciones ejecutivas, lenguaje y habilidades matemáticas. Rev. Estudios Investigación Psicol. Educ. 9, 073-078. doi: 10.17979/reipe.2015.0.09.577

Roberts, L., Marinis, T., Felser, C., and Clahsen, H. (2007). Antecedent priming at trace positions in children's sentence processing. J. Psycholinguist. Res. 36, 175-188. doi: 10.1007/s10936-006-9038-3

Rojas-Barahona, C. A., Förster, C. E., Moreno-Ríos, S., and McClelland, M. M. (2015). Improvement of working memory in preschoolers and its impact on early literacy skills: a study in deprived communities of rural and urban areas. Early Educ. Dev. 26, 871-892. doi: 10.1080/10409289.2015.1036346

Rutherford, B. R., Wager, T. D., and Roose, S. P. (2010). Expectancy and the treatment of depression: a review of experimental methodology and effects on patient outcome. Current Psychiatry Rev. 6, 1-10. doi: 10.2174/1573400107905 96571

Simons, D. J., Boot, W. R., Charness, N., Gathercole, S. E., Chabris, C. F., Hambrick, D. Z., et al. (2016). Do "brain-training" programs work? Psychol. Sci. Public Interest 17, 103-186. doi: 10.1177/15291006166 61983

Slevc, L. R. (2011). Saying what's on your mind: working memory effects on sentence production. J. Exp. Psychol. 37:1503. doi: 10.1037/a0024350

Slot, P. L., and Von Suchodoletz, A. (2018). Bidirectionality in preschool children's executive functions and language skills: is one developing skill the better predictor of the other? Early Child. Res. Q. 42, 205-214. doi: 10.1016/j.ecresq.2017.10.005

Sohlberg, M. M., and Mateer, C. A. (1987). Effectiveness of an attention-training program. J. Clin. Exp. Neuropsychol. 9, 117-130. doi: 10.1080/016886387084 05352

Spreij, L. A., Visser-Meily, J., van Heugten, C. M., and Nijboer, T. C. (2014). Novel insights into the rehabilitation of memory post acquired brain injury: a systematic review. Front. Hum. Neurosci. 8:993. doi: 10.3389/fnhum.2014. 00993

Swanson, H. L., Zheng, X., and Jerman, O. (2009). Working memory, short-term memory, and reading disabilities: a selective meta-analysis of the literature. J. Learn. Disabil. 42, 260-287. doi: 10.1177/00222194093 31958

Tang, Y. Y., Ma, Y., Wang, J., Fan, Y., Feng, S., Lu, Q., et al. (2007). Short-term meditation training improves attention and self-regulation. Proc. Natl. Acad. Sci. U.S.A. 104, 17152-17156. doi: 10.1073/pnas.07076 78104

Valk, S. L., Bernhardt, B. C., Trautwein, F. M., Böckler, A., Kanske, P., Guizard, N., et al. (2017). Structural plasticity of the social brain: differential change after socio-affective and cognitive mental training. Sci. Adv. 3:e1700489. doi: 10.1126/sciadv.17 00489

van Heugten, C. M., Ponds, R. W., and Kessels, R. P. (2016). Brain training: hype or hope? Neuropsychol. Rehabil. 26, 639-644. doi: 10.1080/09602011.2016.1186101 
Wang, P., Liu, H. H., Zhu, X. T., Meng, T., Li, H. J., and Zuo, X. N. (2016). Action video game training for healthy adults: a meta-analytic study. Front. Psychol. 7:907. doi: 10.3389/fpsyg.2016.00907

Weiland, C., Barata, M. C., and Yoshikawa, H. (2014). The cooccurring development of executive function skills and receptive vocabulary in preschool-aged children: a look at the direction of the developmental pathways. Infant Child Dev. 23, 4-21. doi: 10.1002/ic d. 1829

Wilson, B. A. (1997). Cognitive rehabilitation: how it is and how it might be. J. Int. Neuropsychol. Soc. 3, 487-496. doi: 10.1017/S1355617797 004876

Woodard, K., Pozzan, L., and Trueswell, J. C. (2016). Taking your own path: individual differences in executive function and language processing skills in child learners. J. Exp. Child Psychol. 141, 187-209. doi: 10.1016/j.jecp.2015.08.005
Zeiss, A. M., Lewinsohn, P. M., and Muñoz, R. F. (1979). Nonspecific improvement effects in depression using interpersonal skills training, pleasant activity schedules, or cognitive training. J. Consult. Clin. Psychol. 47:427. doi: 10.1037/0022-006X.47.3.427

Conflict of Interest: The authors declare that the research was conducted in the absence of any commercial or financial relationships that could be construed as a potential conflict of interest.

Copyright $\odot 2021$ Tapia and Dunabeitia. This is an open-access article distributed under the terms of the Creative Commons Attribution License (CC BY). The use, distribution or reproduction in other forums is permitted, provided the original author(s) and the copyright owner(s) are credited and that the original publication in this journal is cited, in accordance with accepted academic practice. No use, distribution or reproduction is permitted which does not comply with these terms. 